\title{
Black hole accretion: theoretical limits and observational implications
}

\author{
Dominikus Heinzeller, ${ }^{1,2}$ Wolfgang J. Duschl, ${ }^{1,2,3}$ Shin Mineshige ${ }^{4}$ \\ and Ken Ohsuga ${ }^{5}$ \\ ${ }^{1}$ Zentrum für Astronomie Heidelberg, Institut für Theoretische Astrophysik, \\ Heidelberg, Germany \\ ${ }^{2}$ Institut für Theoretische Physik und Astrophysik, Universität Kiel, Kiel, Germany \\ ${ }^{3}$ Steward Observatory, The University of Arizona, Tucson, AZ 85721, USA \\ ${ }^{4}$ Yukawa Institute for Theoretical Physics, Kyoto University, Sakyo-ku, Kyoto, Japan \\ ${ }^{5}$ Department of Physics, Rikkyo University, Toshimaku, Tokyo, Japan
}

email: hd, wjd@astrophysik.uni-kiel.de,minesige@yukawa.kyoto-u.ac.jp, k_ohsuga@rikkyo.ac.jp

\begin{abstract}
Recently, the issue of the role of the Eddington limit in accretion discs became a matter of debate. While the classical (spherical) Eddington limit is certainly an over-simplification, it is not really clear how to treat it in a flattened structure like an accretion disc. We calculate the critical accretion rates and resulting disc luminosities for various disc models corresponding to the classical Eddington limit by equating the attractive and repulsive forces locally. We also discuss the observational appearance of such highly accreting systems by analyzing their spectral energy distributions. Our calculations indicate that the allowed mass accretion rates differ considerably from what one expects by applying the Eddington limit in its classical form, while the luminosities only weakly exceed their classical equivalent. Depending on the orientation of the disc relative to the observer, mild relativistic beaming turns out to have an important influence on the disc spectra. Thus, possible super-Eddington accretion, combined with mild relativistic beaming, supports the idea that ultraluminous X-ray sources host stellar mass black holes and accounts partially for the observed high temperatures of these objects.
\end{abstract}

Keywords. accretion, accretion discs - radiative transfer - galaxies: active - galaxies: nuclei

\section{Introduction}

With the constant improvement of observational techniques in the past decades, more and more detailed information about accretion disc systems could be gained. In particular, a large number of ultraluminous X-ray sources (ULXs) have been discovered (Fabbiano 1989), imposing a severe problem upon the existing general idea of accretion disc systems: With a bolometric luminosity exceeding $10^{39} \mathrm{erg} \mathrm{s}^{-1}$, at least some of them show relatively low radiation temperatures $(\sim 0.1 \mathrm{keV})$. These systems have been suggested to be intermediate mass black hole (IMBH), sub-Eddington accretion disc systems (Roberts, Warwick, Ward et al. (2005)). However, large samples of ULXs from recent observational data reveal that a distinct class exists, showing higher temperatures sometimes exceeding $1 \mathrm{keV}$ - than can be explained by IMBHs (Mizuno, Ohnishi, Kubota et al. (1999)). In contrast, stellar mass black holes accreting above their Eddington limit can account for these sources (Watarai, Mizuno \& Mineshige (2001)).

In particular, the applicability of the classical Eddington limit is an important point in this discussion: Similar to the stellar case, disc accretion may be limited by radiation pressure, counteracting gravity and viscous dissipation. While in the stellar case, we are dealing with an approximately spherically symmetric, i.e., 1-dimensional situation, 
discs require an - at least - 2-dimensional treatment. The stellar Eddington limit implies several assumptions, which do not apply for the disc case, though it is not clear a priori to what degree a proper treatment will alter the resulting numbers.

In our work, we calculate the local analog to the Eddington limit for a classical thin disc model of the Shakura \& Sunyaev (1973) variety and for a slim disc model of the Abramowicz, Czerny, Lasota et al. (1988) type (Sect. 2). In both descriptions, a standard $\alpha$-viscosity is applied, therefore restricting the models to the non-selfgravitating case. We also investigate the spectral energy distribution of supercritical accretion flows in the stellar mass black hole case, based on the radiation hydrodynamic (RHD) simulations computed by Ohsuga, Mori, Nakamoto et al. (2005) (Sect. 3).

\section{Black hole accretion and the Eddington limit}

The physical reasoning for the Eddington limit is an equilibrium of the attractive force $F_{\mathrm{g}}$ and the repelling force $F_{\mathrm{r}}$. The original stellar Eddington limit relies on several assumptions: (1) spherical symmetry of the system; (2) isotropic radiation; (3) homogeneous degree of ionisation; (4) Thomson scattering as the sole source of opacity; (5) negligible gas pressure; (6) no relativistic effects; and (7) no time dependence (stationarity). To investigate if these assumptions are an oversimplification in the disc case, we relinquish the approximations (1)-(5), but keep the approximation of non-relativistic stationarity for our calculation.

We assume azimuthal symmetry in the following. Therefore, one can define two different "Eddington limits", corresponding to the equilibria in the vertical $(z)$ and radial $(s)$ direction. (We use a cylindrical coordinate system $\{s, \varphi, z\}$ with the distance $r$ to the origin, $r^{2}=s^{2}+z^{2}$.) For a rotating viscous disc, further contributions to the total force have to be included in the radial direction:

$$
F_{\text {tot }}^{(z)}(s)=F_{\mathrm{r}}^{(z)}(s)-F_{\mathrm{g}}^{(z)}(s)=0, \quad F_{\text {tot }}^{(s)}(s)=F_{\mathrm{r}}^{(s)}(s)-F_{\mathrm{g}}^{(s)}(s)+\ldots=0 .
$$

In the following, as an example, we calculate the maximum amount of matter $\dot{M}_{\text {crit }}$ that can be accreted towards the central object in the case of a stellar mass black hole, $M=10 M_{\odot}$, for non-selfgravitating discs with an $\alpha$-parameter of 0.1 . For both the thin and the slim disc case, we use two descriptions for the opacity: Firstly, simple Thomson scattering is applied: $\kappa_{\mathrm{es}}=0.4 \mathrm{~cm}^{2} \mathrm{~g}^{-1}$. Secondly, we use an interpolation formula, $\kappa=\kappa_{\text {in }}$ (Gail, priv. comm.), which accounts for further contributions like bound-free and free-free absorption. For a detailed description of $\kappa_{\text {in }}$ see Heinzeller \& Duschl (2006).

We refer our results to the classical Eddington-limit, which in the disc case with a torque-free boundary condition at the disc's inner radius $s_{\mathrm{i}}=3 r_{\mathrm{g}}=6 G M / \mathrm{c}^{2}$ is given by $L_{\mathrm{E}}=4 \pi c G M / \kappa_{\mathrm{es}}=1.2 \cdot 10^{39} \mathrm{erg} / \mathrm{s}$, and $\dot{M}_{\mathrm{E}}=8 \pi c s_{\mathrm{i}} / \kappa_{\mathrm{es}}=2.6 \cdot 10^{-7} M_{\odot} /$ a. Detailed calculations reveal that the vertical limit sets severer conditions on the critical accretion rates and corresponding luminosities than the radial limit in both the thin and the slim disc model. We therefore concentrate on the results for the vertical limit in the following.

Figure 1 shows the results for the critical accretion rates $\dot{M}_{\text {crit }}$ in the vertical Eddington limit as a function of the radial distance $s$ from the central object. In the slim disc case, one free parameter remains to be determined by hand: the ratio of the local height $h$ of the disc to the local radial distance $s$. Since the slim disc model is valid for $h / s \leqslant 1$, we plot the results for the two cases $(h / s)_{\max }=1.0$ and $(h / s)_{\max }=0.5$.

Obviously, the critical accretion rates are no longer given by a global quantity like $\dot{M}_{E}$ : they depend on the radius $\propto s^{1.2 \ldots 1.9}$. While the opacity has a strong influence on the results $\left(\dot{M}_{\text {crit }} \propto \kappa^{-1}\right)$ for low accretion rates, it becomes more and more unimportant 

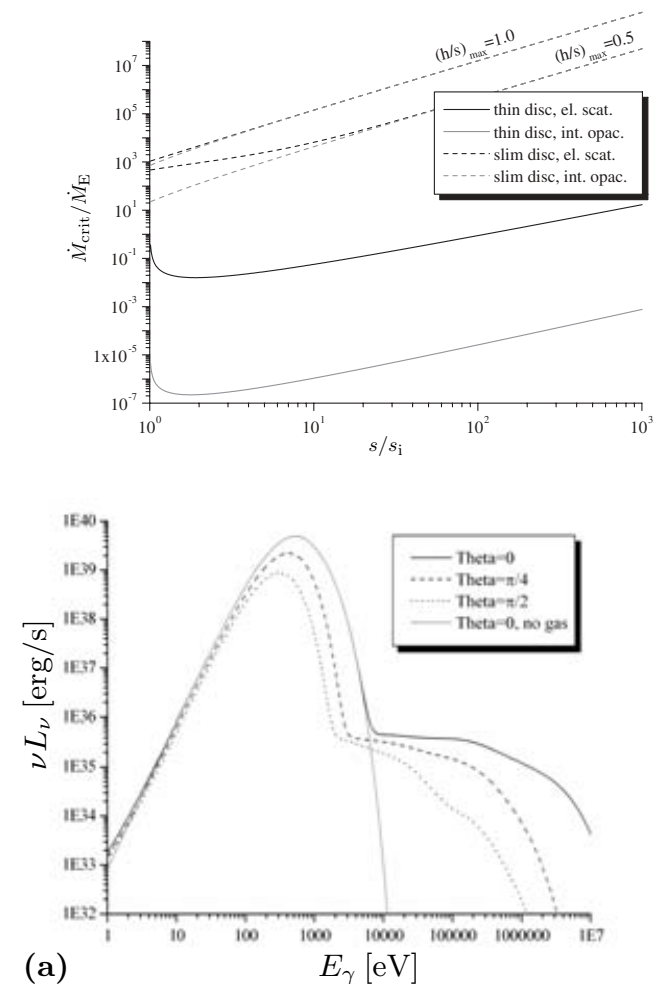

Figure 1. Critical local accretion rates in the vertical Eddington limit as a function of the radial distance $s$ from the central object. For slim discs, $(h / s)_{\max }=1.0$ in the upper and $(h / s)_{\max }=0.5$ in the lower case. Black lines correspond to Thomson scattering, $\kappa=\kappa_{\mathrm{es}}$, gray lines to interpolated opacities, $\kappa=\kappa_{\text {in }}$.

Figure 2. (a) Spectral energy distribution for various inclination angles $\Theta$; (b) Comparison of total intensity, average photon energy and total photon number for $\Theta=0 \ldots \pi / 2$, normalized to the corresponding values at $\pi / 2$.

in the slim disc model for high accretion rates. This is due to the fact that advection dominates the energy transport in this regime. As can be seen from the results for thin discs, the inner boundary condition plays a crucial role in determining the maximum amount of matter which finally reaches the central black hole. For slim discs, the critical accretion rates may reach up to $10^{3} \dot{M}_{\mathrm{E}}$, while at the same time the luminosity stays close to the classical limit, $L \approx 20 L_{E}$ (Watarai, Fukue, Takeuchi et al. (2000)). Thus, highly super-Eddington accretion comes along with only mildly super-Eddington luminosities.

\section{Spectral energy distribution of super-Eddington flows}

In the second part, we investigate the observational appearance of super-Eddington accretion discs. This work is based on 2D RHD simulations, which are presented in detail in Ohsuga, Mori, Nakamoto et al. (2005). In a spherical computational box of $500 R_{\mathrm{G}}$ radius, matter is injected continuously in the disc plane $x-y$ around a $10 M_{\odot}$ black hole at high rates $\left(\dot{M}=10^{3} \dot{M}_{\mathrm{E}}\right)$. Again, the viscosity is parameterized by $\alpha=0.1$. For the energy transport equation, radiation and advection are taken into account, as well as photon trapping effects (Begelman (1978)). We calculate the spectral energy distribution of this system for various inclination angles $\Theta$ between 0 (face-on) and $\pi / 2$ (edge-on). For details about the computation, we refer the reader to Heinzeller, Mineshige \& Ohsuga (2006). The resulting SEDs for inclination angles $0, \pi / 4$ and $\pi / 2$ are shown in Fig. 2(a).

While in the Rayleigh-Jeans part of the spectrum no difference between the orientations of the system can be seen, the peak intensity region around $1 \mathrm{keV}$ is shifted towards higher 
photon energies and higher luminosities for lower inclination angles. For all orientations, a plateau-like structure in the high-energy part of the spectrum can be observed, although this feature is more prominent for the face-on case. Its origin lies in the emission of the hot gas in the vicinity of the black hole: when calculating the spectrum for $\Theta=0$ while neglecting gas emission and absorption, this plateau disappears.

To outline the orientation effects, we compare in Fig. 2(b) the total intensity emitted by the system for inclination angles $\Theta=\{0 \ldots \pi / 2\}$. For small inclinations, the intensity rises by a factor of up to 6.4 for $\Theta=0$; this is due to both an enhanced average photon energy and an increased total photon number. We identify this behavior with mild relativistic beaming. When fitting the peak intensity region with a black body spectrum, we derive temperatures between $1.1 \cdot 10^{6} \mathrm{~K}$ for $\Theta=\pi / 2$ and $1.7 \cdot 10^{6} \mathrm{~K}$ for $\Theta=0$.

\section{Conclusions}

The above investigations allow us to draw several conclusions on black hole accretion processes. Firstly, we show that the classical Eddington limit can not be applied in discs. Contrary, the critical accretion rates become a local quantity, depending on the distance from the central object. Thereby, the inner disc region and inner boundary hold the key position in determining the final amount of matter that can be accreted by the central object.

We find that super-Eddington accretion is possible with accretion rates up to $10^{3} \dot{M}_{\mathrm{E}}$, corresponding to slightly super-Eddington luminosities up to $20 L_{\mathrm{E}}$. Since the bottleneck for accretion lies in the inner region of the disc, our results may also correlate to the question on the origin of outflows and jets. Secondly, our work on the spectral energy distribution of such super-Eddington accreting systems shows that for a proper interpretation of the spectrum, the combined system of the disc and its surroundings has to be taken into account.

Features like the high-energy plateau can not be reproduced by considering the accretion disc only. Also, we find that mild relativistic beaming becomes important for small inclination angles, leading to higher luminosities and also higher temperatures. However, these temperatures are still too low to account for the observed ULX sources which sometimes reach temperatures up to $10^{7} \mathrm{~K}$. This may be due to the lack of Comptonization effects in our calculations: Socrates, Davis \& Blaes (2004), for example, showed that turbulent Comptonization produces a significant contribution to the far-UV and X-ray emission of black hole accretion discs. Thus, the inclusion of Comptonization effects will be a primary goal in our future investigations.

\section{References}

Abramowicz, M. A., Czerny, B., Lasota, J.-P. \& Szuzkiewicz, E. 1988, ApJ, 332, 646

Begelman, M. C. 1978, MNRAS, 184, 53

Fabbiano, G. 1989, ARA\&A, 27, 87

Heinzeller, D. \& Duschl, W. J. 2006, to appear in MNRAS

Heinzeller, D., Mineshige, S. \& Ohsuga, K. 2006, to appear in MNRAS

Mizuno, T., Ohnishi, T., Kubota, A., Makishima, K. \& Tashiro, M. 1999, PASJ, 51, 663

Ohsuga, K., Mori, M., Nakamoto, T. \& Mineshige, S. 2005, ApJ, 628, 368

Roberts, T. P., Warwick, R. S., Ward, M. J., Goad, M. R. \& Jenkins, L. P. 2005, MNRAS, 357, 1363

Shakura, N. I. \& Sunyaev, R. A. 1973, A\&A, 24, 337

Socrates, A., Davis, S. W. \& Blaes, O. 2004, ApJ, 601, 405

Watarai, K.-Y., Fukue, J., Takeuchi, M. \& Mineshige, S. 2000, PASJ, 52, 133

Watarai, K.-Y., Mizuno, T. \& Mineshige, S. 2001, ApJ, 549, L77 
Richard Mushotzky: Two questions: first, have you done a stability analysis? And second, temperatures seem to be low compared to ULX...

Dominikus Heinzeller: 1. No, we have not performed a stability analysis yet. Also the time-dependency of these highly accreting systems has not been studied to date, but it is one of primary goals for the future work. 2. Indeed, our temperatures are too low to explain the highest observed temperatures of ULXs. However, Comptonization effects have not been included in the radiative transfer calculations. We expect that Comptonization will increase the effective temperatures, mostly for low inclination angles (i.e. a face-on view).

ANDREW LAWRENCE: Isn't the simplest way to explain super-Eddington sources to abandon the assumption that they are in steady state? In other words, what if they are in outburst?

Dominikus Heinzeller: May be - or may not. I can't answer this at the present, but investigations have to reveal if the duration of outburst lasts sufficiently long to explain the lifetime of super-Eddington states in these sources. 


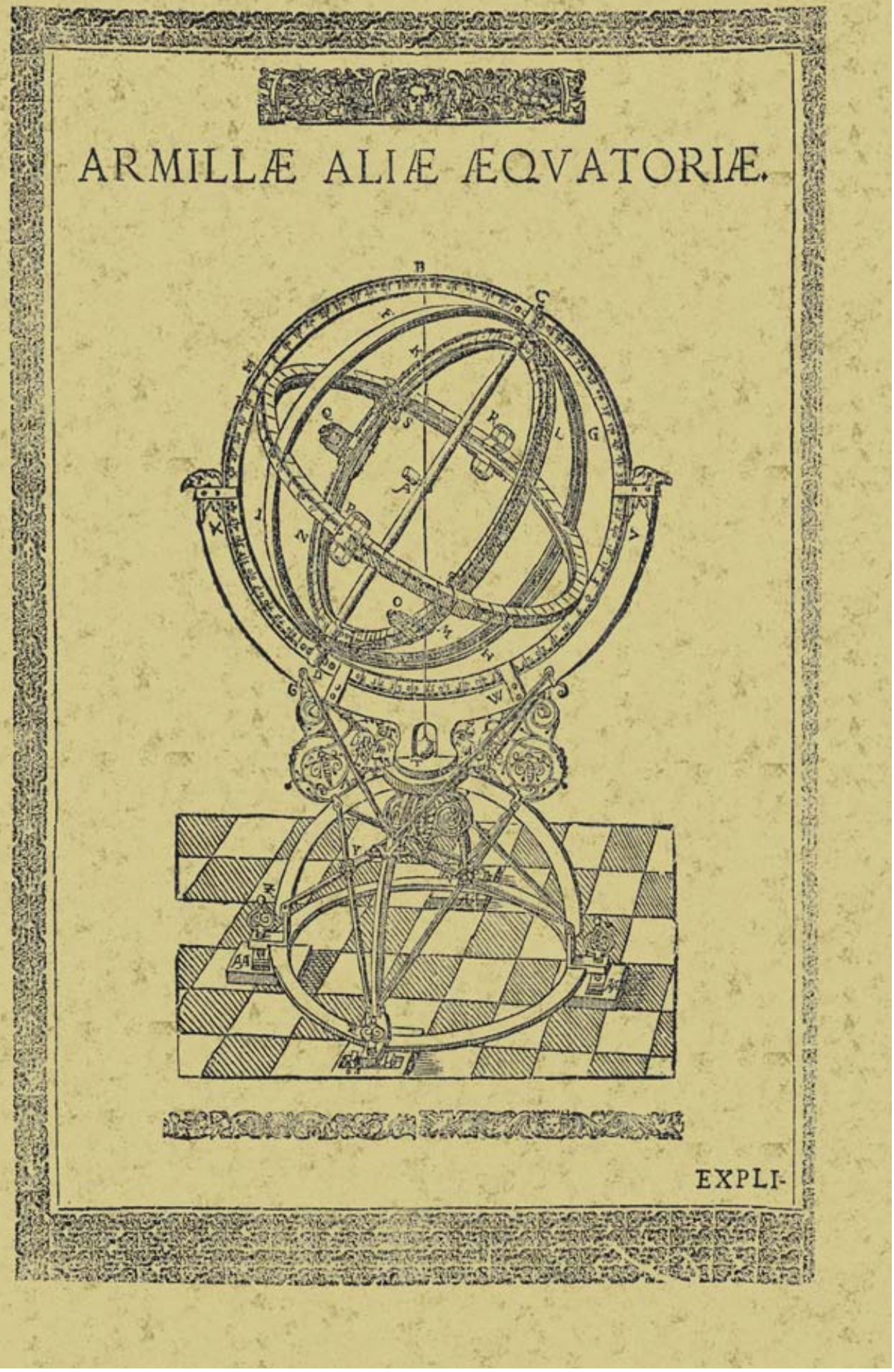

